\title{
ON THE NUMBER OF SOLUTIONS OF A THIRD-ORDER BOUNDARY VALUE PROBLEM
}

\author{
EVA ROVDEROVÁ
}

\begin{abstract}
This paper deals with the number of solutions of the third-order boundary value problem $y^{\prime \prime \prime}=f\left(t, y, y^{\prime}, y^{\prime \prime}\right), y(0)=A_{0}, y^{\prime}(0)=A_{1}$, $y^{\prime \prime}(T)=B$. This number of solutions is investigated in connection with the number of zeros of a solution for the corresponding variational problem.
\end{abstract}

\section{INTRODUCTION}

The aim of this paper is to study the number of solutions for the two-point boundary value problem (in short BVP)

$$
\begin{aligned}
& y^{\prime \prime \prime}=f\left(t, y, y^{\prime}, y^{\prime \prime}\right), \\
& y(0)=A_{0}, \quad y^{\prime}(0)=A_{1}, \\
& y^{\prime \prime}(T)=B,
\end{aligned}
$$

where $f, \frac{\partial f}{\partial y}, \frac{\partial f}{\partial y^{\prime}}, \frac{\partial f}{\partial y^{\prime \prime}}$ are continuous functions on $[0, T] \times R^{3}$, and $A_{0}$, $A_{1}, B$ are given real numbers.

Some problems of regulation and control of some actions by a control level or by a signal reduce to solving the third-order equations [8]. Another application of the third-order differential equations is encountered in the control of a flying apparatus in cosmic space [7].

The following initial value problem

$$
\begin{aligned}
z^{\prime \prime \prime}= & \frac{\partial f}{\partial y^{\prime \prime}}\left(t, \xi(t), \xi^{\prime}(t), \xi^{\prime \prime}(t)\right) z^{\prime \prime}+\frac{\partial f}{\partial y^{\prime}}\left(t, \xi(t), \xi^{\prime}(t), \xi^{\prime \prime}(t)\right) z^{\prime} \\
& +\frac{\partial f}{\partial y}\left(t, \xi(t), \xi^{\prime}(t), \xi^{\prime \prime}(t)\right) z,
\end{aligned}
$$

$$
z(0)=0, \quad z^{\prime}(0)=0, \quad z^{\prime \prime}(0)=1
$$

is the corresponding variational problem, where $\xi$ is a solution of (1), (2), (3). The differential equation (4) is the variation equation for equation (1) along the

Received by the editors June 22, 1993; originally communicated to the Proceedings of the AMS by Hal L. Smith.

1991 Mathematics Subject Classification. Primary 34B15.

Key words and phrases. Third-order nonlinear differential equations, boundary conditions, variation equation, index of a solution. 
solution $\xi$. We will show that the number of solutions of (1), (2), (3) depends on the number of zeros of a solution $z$ of (4), (5).

\section{Main Results}

Definition 1. Let $\xi$ be a solution of (1), (2), (3) and $z$ be a corresponding solution of (4), (5). Then the function $\xi:[0, T] \rightarrow R$ has the index $m(m=$ $0,1,2, \ldots)$ if and only if $z(t)$ has $m$ zeros $0<t_{1}<t_{2}<\cdots<t_{m}<T$.

Throughout the whole paper we will suppose that:

(i) there exists a solution $\xi$ of (1), (2), (3) with index $m \geq 3$;

(ii) there exists a solution $\eta$ of $(1),(2)$ on $[0, T]$ such that $\xi(t)<\eta(t)$ for all $t \in(0, T]$;

(iii) all solutions $y$ of $(1),(2)$ for which $\xi^{\prime \prime}(0) \leq y^{\prime \prime}(0) \leq \eta^{\prime \prime}(0)$ extend to $[0, T]$

(iv) the function $f$ fulfills the conditions:

$$
\begin{gathered}
\frac{\partial f}{\partial y^{\prime \prime}}\left(t, y, y^{\prime}, y^{\prime \prime}\right) \leq 0 \\
2 \frac{\partial f}{\partial y}\left(t, y, y^{\prime}, y^{\prime \prime}\right)-\frac{d}{d t}\left[\frac{\partial f}{\partial y^{\prime}}\left(t, y, y^{\prime}, y^{\prime \prime}\right)\right] \\
+\frac{\partial f}{\partial y^{\prime \prime}}\left(t, y, y^{\prime}, y^{\prime \prime}\right) \cdot \frac{\partial f}{\partial y^{\prime}}\left(t, y, y^{\prime}, y^{\prime \prime}\right) \leq 0 \\
\frac{\partial f}{\partial y^{\prime}}\left(t, y, y^{\prime}, y^{\prime \prime}\right) \leq 0
\end{gathered}
$$

for all $\left(t, y, y^{\prime}, y^{\prime \prime}\right) \in[0, T] \times R^{3}$ and

$$
2 \frac{\partial f}{\partial y}(t, \cdot, \cdot, \cdot)-\frac{d}{d t}\left[\frac{\partial f}{\partial y^{\prime}}(t, \cdot, \cdot, \cdot)\right]+\frac{\partial f}{\partial y^{\prime \prime}}(t, \cdot, \cdot, \cdot) \cdot \frac{\partial f}{\partial y^{\prime}}(t, \cdot, \cdot, \cdot)
$$

is not identically zero on $[0, T]$ for every fixed $\left(y, y, y^{\prime \prime}\right) \in R^{3}$.

Lemma 1. Let $\eta$ be a solution of (1), (2) having the property (ii). Then $\xi^{\prime \prime}(0)<$ $\eta^{\prime \prime}(0)$.

Proof. If $\xi^{\prime \prime}(0)=\eta^{\prime \prime}(0)$, then $\xi$, $\eta$ satisfy the same initial value problem for (1). From the Carathéodory local uniqueness theorem we get that $\xi=\eta$ on $[0, \delta)$ which contradicts (ii). The condition $\xi^{\prime \prime}(0)>\eta^{\prime \prime}(0)$ also contradicts (ii).

Lemma 2. Consider the differential equation

$$
z^{\prime \prime \prime}+p(t) z^{\prime \prime}+q(t) z^{\prime}+r(t) z=0
$$

where $p, r$ are continuous functions and $q$ is a continuously differentiable function on $[0, T]$. Let further

$$
p(t) \geq 0, \quad 2 r(t)-q^{\prime}(t)-p(t) q(t) \geq 0
$$

and the sign of the equality in the second inequality does not hold on any subinterval of $[0, T]$. Let $z$ be a solution of $(6)$ such that $z\left(t_{0}\right)=z^{\prime}\left(t_{0}\right)=0, z^{\prime \prime}\left(t_{0}\right) \neq 0$ where $t_{0} \in[0, T]$. 
Then the solution $z$ does not have any null-point on the interval $[0, T]$ to the left of $t_{0}\left(\left[2\right.\right.$, Lemma 1]). Moreover, if $q(t) \geq 0$ on $[0, T]$ and $z^{\prime}(T)=0$, then $z(T) z^{\prime \prime}(T)<0$.

Proof. We will prove only the second statement. From the equation (6) we get the following identity

$$
\begin{aligned}
& \left\{\left[z^{\prime 2}(t)-2 z(t) z^{\prime \prime}(t)-q(t) z^{2}(t)\right] e^{\int_{0}^{t} p(s) d s}\right\}^{\prime} \\
& \quad=\left\{p(t) z^{\prime 2}(t)+\left[2 r(t)-q^{\prime}(t)-p(t) q(t)\right] z^{2}(t)\right\} e^{\int_{0}^{t} p(s) d s} .
\end{aligned}
$$

Let us denote

$$
F(t)=\left[z^{\prime 2}(t)-2 z(t) z^{\prime \prime}(t)-q(t) z^{2}(t)\right] e^{\int_{0}^{t} p(s) d s} .
$$

From the assumption (7) we have that $F^{\prime}(t) \geq 0$ and $F^{\prime}(t)$ is not identically zero on any subinterval of $[0, T]$. Hence $F(t)$ is strictly increasing on $[0, T]$ and so $F(0)<F(T)$. After substitution we have

$$
F(0)=0, \quad F(T)=\left[z^{\prime 2}(T)-2 z(T) z^{\prime \prime}(T)-q(T) z^{2}(T)\right] e_{0}^{t} p(s) d s
$$

and so

$$
\begin{aligned}
& 0<\left[-2 z(T) z^{\prime \prime}(T)-q(T) z^{2}(T)\right] e^{\int_{0}^{T} p(s) d s} \\
& 0<-2 z(T) z^{\prime \prime}(T)-q(T) z^{2}(T) \\
& 2 z(T) z^{\prime \prime}(T)<-q(T) z^{2}(T) \leq 0
\end{aligned}
$$

Hence the proof is complete.

Remark 1. From Lemma 2 and (iv) it follows, that if $z$ is a solution of (4), (5), then all its zeros (if they exist) are simple in $(0, T]$.

Let us denote $\Lambda_{0}=\xi^{\prime \prime}(0), \Lambda_{1}=\eta^{\prime \prime}(0)$. The set $\left\{A_{0}\right\} \times\left\{A_{1}\right\} \times\left[\Lambda_{0}, \Lambda_{1}\right]$ is compact in $R^{3}$ and so the following lemma holds:

Lemma 3 [3, Lemma 5.5]. Let $S$ be the set of all solutions of (1), (2) such that $\xi^{\prime \prime}(0) \leq y^{\prime \prime}(0) \leq \eta^{\prime \prime}(0)$. Then the set $S$ is a compact in $C^{2}([0, T], R)$.

Theorem 1. Let $y(t, \lambda)$ be a solution of the initial value problem

$$
\begin{aligned}
& y^{\prime \prime \prime}=f\left(t, y, y^{\prime}, y^{\prime \prime}\right), \\
& y(0)=A_{0}, \quad y^{\prime}(0)=A_{1}, \\
& y^{\prime \prime}(0)=\lambda,
\end{aligned}
$$

where $\lambda \in\left[\Lambda_{0}, \Lambda_{1}\right]$. Let

$$
z(t, \lambda)=\frac{y(t, \lambda)-\xi(t)}{\lambda-\Lambda_{0}}
$$

Then there exists $a \delta>0$ such that for all $\lambda \in\left(\Lambda_{0}, \Lambda_{0}+\delta\right)$ the function $z(t, \lambda)$ has at least $m$ simple zeros $0<t_{1}(\lambda)<t_{2}(\lambda)<\cdots<t_{m}(\lambda)<T$. Moreover, all zeros of $z(t, \lambda)$ (if they exist) are simple in $(0, T]$ for $\lambda \in\left(\Lambda_{0}, \Lambda_{1}\right]$.

Proof. We use the theorem about the continuous dependence on initial conditions [3, Theorem 6.2]. Let $\lambda \in\left(\Lambda_{0}, \Lambda_{1}\right]$. Then

$$
\begin{aligned}
& \lim _{\lambda \rightarrow \Lambda_{0}} \frac{\partial^{j} y(t, \lambda)}{\partial t^{j}}=\xi^{(j)}(t)=\frac{\partial^{j} y\left(t, \Lambda_{0}\right)}{\partial t^{j}}, \\
& \lim _{\lambda \rightarrow \Lambda_{1}} \frac{\partial^{j} y(t, \lambda)}{\partial t^{j}}=\eta^{(j)}(t)=\frac{\partial^{j} y\left(t, \Lambda_{1}\right)}{\partial t^{j}}
\end{aligned}
$$


uniformly on $[0, T]$ for $j=0,1,2$. The function $z(t, \lambda)$ defined by (9) is the solution of the initial value problem

$$
\begin{gathered}
z^{\prime \prime \prime}=g_{2}(t, \lambda) z^{\prime \prime}+g_{1}(t, \lambda) z^{\prime}+g_{0}(t, \lambda) z \\
z(0, \lambda)=\frac{y(0, \lambda)-\xi(0)}{\lambda-\Lambda_{0}}=\frac{A_{0}-A_{0}}{\lambda-\Lambda_{0}}=0, \\
z^{\prime}(0, \lambda)=\frac{y^{\prime}(0, \lambda)-\xi^{\prime}(0)}{\lambda-\Lambda_{0}}=\frac{A_{1}-A_{1}}{\lambda-\Lambda_{0}}=0, \\
z^{\prime \prime}(0, \lambda)=\frac{y^{\prime \prime}(0, \lambda)-\xi^{\prime \prime}(0)}{\lambda-\Lambda_{0}}=\frac{\lambda-\Lambda_{0}}{\lambda-\Lambda_{0}}=1
\end{gathered}
$$

where $\left(1=\frac{d}{d t}\right)$

$$
\begin{aligned}
& g_{2}(t, \lambda)=\int_{0}^{1} \frac{\partial f}{\partial y^{\prime \prime}}\left[t, y\left(t, \Lambda_{0}\right), y^{\prime}\left(t, \Lambda_{0}\right), u_{2}(t, s, \lambda)\right] d s, \\
& g_{1}(t, \lambda)=\int_{0}^{1} \frac{\partial f}{\partial y^{\prime}}\left[t, y\left(t, \Lambda_{0}\right), u_{1}(t, s, \lambda), y^{\prime \prime}(t, \lambda)\right] d s \\
& g_{0}(t, \lambda)=\int_{0}^{1} \frac{\partial f}{\partial y}\left[t, u_{0}(t, s, \lambda), y^{\prime}(t, \lambda), y^{\prime \prime}(t, \lambda)\right] d s,
\end{aligned}
$$

and

$$
u_{j}(t, s, \lambda)=\xi^{(j)}(t)+s \cdot\left[\frac{\partial^{j} y(t, \lambda)}{\partial t^{j}}-\xi^{(j)}(t)\right], \quad 0 \leq s \leq 1, \quad j=0,1,2 .
$$

Furthermore,

(10)

$$
g_{2}(t, \lambda) \leq 0, \quad g_{1}(t, \lambda) \leq 0, \quad 2 g_{0}(t, \lambda)-\frac{d}{d t} g_{1}(t, \lambda)+g_{2}(t, \lambda) g_{1}(t, \lambda) \leq 0
$$

and the sign of the equality in the last inequality does not hold on any subinterval of $[0, T]$. From Lemma 2 we have that all zeros of $z(t, \lambda)$ are simple in $(0, T]$ for $\lambda \in\left(\Lambda_{0}, \Lambda_{1}\right]$. It is easy to see that the initial value problem $\left(4^{*}\right),(5) \rightarrow(4)$, (5) as $\lambda \rightarrow \Lambda_{0}$ and therefore $\partial y\left(t, \Lambda_{0}\right) / \partial \lambda=z(t)$ on $[0, T]$. Furthermore,

$$
\lim _{\lambda \rightarrow \Lambda_{0}} \frac{\partial^{j} z(t, \lambda)}{\partial t^{j}}=z^{(j)}(t)
$$

uniformly on $[0, T]$. Since $z(t)$ has $m$ simple zeros in $(0, T)$ and $z(t, \lambda)$ tends to $z(t)$ uniformly, there exists a $\delta>0$ such that for all $\lambda \in\left(\Lambda_{0}, \Lambda_{0}+\delta\right)$ the function $z(t, \lambda)$ has $m$ simple zeros $0<t_{1}(\lambda)<t_{2}(\lambda)<\cdots<t_{m}(\lambda)<T$.

Remark 2. Later in Theorem 3 we will apply the shooting method on the problem (1), (2), (8); i.e., we will find $\lambda$ such that the solution $y(t, \lambda)$ of $(1),(2)$, (8) will satisfy the boundary condition (3). We have that the problem $\left(4^{*}\right)$, $(5) \rightarrow(4),(5)$ as $\lambda \rightarrow \Lambda_{0}$. Since there exists the advanced theory of the third-order linear differential equations, the variational problem (4), (5) was suitably chosen in order to extend the facts on the problem $\left(4^{*}\right),(5)$. From the definition of function $z(t, \lambda)$ in Theorem 1 we have that for $\lambda$ close to $\Lambda_{0}$ the function $y(t, \lambda)$ intersect $y\left(t, \Lambda_{0}\right) \equiv \xi(t)$ in $m$ distinct points $t_{r}(\lambda)$ in $(0, T), r=1,2, \ldots, m$. On the other side, from the assumption (ii) we have that $y\left(t, \Lambda_{1}\right) \equiv \eta(t)$ does not intersect $y\left(t, \Lambda_{0}\right) \equiv \xi(t)$ in $(0, T]$ and so 
for $\lambda$ close to $\Lambda_{1}$ the function $y(t, \lambda)$ does not intersect $y\left(t, \Lambda_{0}\right)$ in $(0, T]$. Consequently, the functions $t_{r}(\lambda)$ are not defined for all $\lambda \in\left[\Lambda_{0}, \Lambda_{1}\right]$. Let $D_{r} \subset\left[\Lambda_{0}, \Lambda_{1}\right]$ be the interval of definition of the function $t_{r}(\lambda)$. It is clearly that $D_{m} \subset D_{m-1} \subset \cdots \subset D_{1} \subset\left[\Lambda_{0}, \Lambda_{1}\right]$. From the following Lemma 4 we have that $t_{r}(\lambda)$ are continuous functions in their domains of definition $D_{r}$, $r=1,2, \ldots, m$.

Lemma 4 [4, Lemma 4.2, p. 145]. Let $z(t, \lambda)$ be a solution of $\left(4^{*}\right),(5)$, and let $t_{1}(\lambda), t_{2}(\lambda), \ldots, t_{m}(\lambda)$ be the zeros of $z(t, \lambda)$ in $(0, T]$. Then $t_{r}(\lambda)$ are continuous functions of the parameter $\lambda$ in their domains of definition $D_{r}, r=$ $1,2, \ldots, m$.

Theorem 2. Let $t_{1}(\lambda), t_{2}(\lambda), \ldots, t_{m}(\lambda)$ be the zeros of $z(t, \lambda)$. Then

$$
\exists h>0: t_{r+1}(\lambda)-t_{r}(\lambda)>h,
$$

in their domains of definition $D_{r}, r=1,2, \ldots, m-1$.

Proof. We prove the case $r=1$. Let $D=D_{2} \cap D_{1}$ and let $\lambda \in D$. (For $\lambda \notin D$ the theorem is true.) Let us suppose contradiction. Then

$$
\begin{gathered}
\forall \varepsilon>0 \exists \bar{\lambda}=\bar{\lambda}(\varepsilon) \in D: 0<t_{2}(\bar{\lambda})-t_{1}(\bar{\lambda})<\varepsilon \text { i.e. } \\
\exists\left\{\bar{\lambda}_{n}\right\}_{n=1}^{\infty} \in D: t_{2}\left(\bar{\lambda}_{n}\right)-t_{1}\left(\bar{\lambda}_{n}\right) \rightarrow 0 .
\end{gathered}
$$

Let $\lim _{n \rightarrow \infty} \bar{\lambda}_{n}=\lambda^{*}$. Then $t_{2}\left(\lambda^{*}\right)=t_{1}\left(\lambda^{*}\right)$. Since $S$ is a compact in $C^{2}([0, T], R)$ there exists a subsequence of $\left\{y\left(t, \bar{\lambda}_{n}\right)\right\}_{n=1}^{\infty}$ which converges to $y\left(t, \lambda^{*}\right) \in S$ uniformly on $[0, T]$. Hence the function

$$
z\left(t, \lambda^{*}\right)=\frac{y\left(t, \lambda^{*}\right)-\xi(t)}{\lambda^{*}-\Lambda_{0}}
$$

has a double zero in $t_{1}\left(\lambda^{*}\right)=t_{2}\left(\lambda^{*}\right)$, which contradicts Theorem 1 .

Theorem 3 (main result). Let assumptions (i), (ii), (iii), (iv) hold. Then the BVP (1), (2), (3) has at least

$$
\begin{array}{ll}
\frac{m-1}{2}+1 \text { solutions } & \text { if } m \text { is odd }, \\
\frac{m-2}{2}+1 \text { solutions } & \text { if } m \text { is even. }
\end{array}
$$

Proof. From Theorem 1 we have that the function $z(t, \lambda)$ defined by (9) has at least $m$ simple zeros $0<t_{1}(\lambda)<t_{2}(\lambda)<\cdots<t_{m}(\lambda)<T$ for $\lambda \rightarrow \Lambda_{0}$. On the other side,

$$
\lim _{\lambda \rightarrow \Lambda_{1}} z(t, \lambda)=\lim _{\lambda \rightarrow \Lambda_{1}} \frac{y(t, \lambda)-y\left(t, \Lambda_{0}\right)}{\lambda-\Lambda_{0}}=\frac{\eta(t)-\xi(t)}{\Lambda_{1}-\Lambda_{0}}>0 .
$$

Since $z(0, \Lambda)=0$, then $z(t, \lambda)>0$ on $(0, T]$ for $\lambda \rightarrow \Lambda_{1}$, i.e. the zeros of $z(t, \lambda)$ are leaving the interal $(0, T]$ through the point $T$ for $\lambda \rightarrow \Lambda_{1}$. From the continuous dependence of $y(t, \lambda)$ of the parameter $\lambda$ and the fact, that all zeros of $z(t, \lambda)$ (if they exist) are simple, we conclude that

$$
\sup \left\{t_{r}(\lambda): \lambda \in D_{r}\right\}=T, \quad r=1,2, \ldots, m .
$$

Since $t_{r}(\lambda)$ are continuous functions of $\lambda$, there exists

$$
\lambda_{r}=\max \left\{\lambda: t_{r}(\lambda)=T\right\}, \quad r=1,2, \ldots, m,
$$




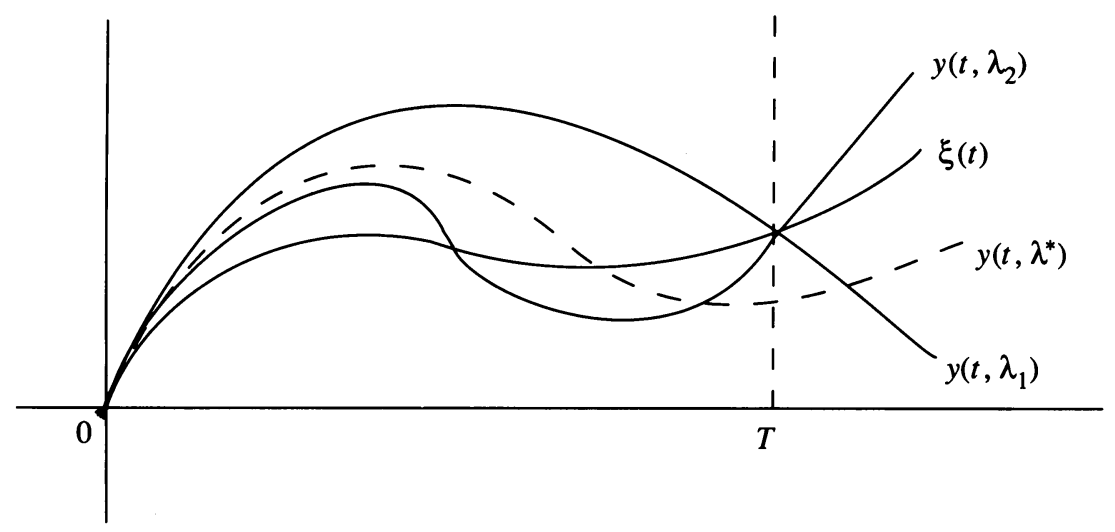

FIGURE 1

and $t_{r}\left(\lambda_{r}\right)=T$ for $r=1,2, \ldots, m$. Theorem 2 implies that

$$
\Lambda_{0}<\lambda_{m}<\lambda_{m-1}<\cdots<\lambda_{2}<\lambda_{1}<\Lambda_{1}
$$

and so

Hence

$$
z\left(T, \lambda_{r}\right)=\frac{y\left(T, \lambda_{r}\right)-\xi(T)}{\lambda_{r}-\Lambda_{0}}=0 .
$$

Since in general

$$
y\left(T, \lambda_{r}\right)=\xi(T), \quad r=1,2, \ldots, m, \ldots
$$

$$
y^{\prime \prime}\left(T, \lambda_{r}\right) \neq \xi^{\prime \prime}(T), \quad r=1,2, \ldots, m, \ldots,
$$

the corresponding functions $y\left(t, \lambda_{r}\right)$ do not have to be the solutions of (1), (2), (3). Thus we make the following analysis:

1. Let us consider the interval $\left(\lambda_{2}, \lambda_{1}\right)$.

From the construction of the numbers $\lambda_{2}, \lambda_{1}$ it follows that

$$
y^{\prime}\left(T, \lambda_{2}\right)>\xi^{\prime}(T)>y^{\prime}\left(T, \lambda_{1}\right) .
$$

Let us define the function

$$
v(\lambda)=y^{\prime}(T, \lambda)-\xi^{\prime}(T) .
$$

Since the function $y^{\prime}$ is continuous in $\lambda$, the function $v$ is continuous and $v\left(\lambda_{2}\right)>0, v\left(\lambda_{1}\right)<0$. Hence there exists a $\lambda^{*} \in\left(\lambda_{2}, \lambda_{1}\right)$ such that $v\left(\lambda^{*}\right)=0$, i.e., $y^{\prime}\left(T, \lambda^{*}\right)=\xi^{\prime}(T)$. Moreover, it is clear (see Figure 1) that $y\left(T, \lambda^{*}\right)<$ $\xi(T)$.

Finally from (10) and Lemma 2 we have

$$
z\left(T, \lambda^{*}\right)<0, \quad z^{\prime}\left(T, \lambda^{*}\right)=0, \quad z^{\prime \prime}\left(T, \lambda^{*}\right)>0 .
$$

2. Let us consider the interval $\left(\lambda_{3}, \lambda_{2}\right)$. Then

$$
y^{\prime}\left(T, \lambda_{3}\right)<\xi^{\prime}(T)<y^{\prime}\left(T, \lambda_{2}\right)
$$

and so $v\left(\lambda_{3}\right)<0, v\left(\lambda_{2}\right)>0$. Hence there exists a $\lambda^{* *} \in\left(\lambda_{3}, \lambda_{2}\right)$ such that $v\left(\lambda^{* *}\right)=0$, i.e., $y^{\prime}\left(T, \lambda^{* *}\right)=\xi(T)$. Obviously, $y\left(T, \lambda^{* *}\right)>\xi(T)$. Again from (10) and Lemma 2 we get

$$
z\left(T, \lambda^{* *}\right)>0, \quad z^{\prime}\left(T, \lambda^{* *}\right)=0, \quad z^{\prime \prime}\left(T, \lambda^{* *}\right)<0 .
$$


So we have found $\lambda^{*}, \lambda^{* *} \in\left(\lambda_{3}, \lambda_{1}\right)$ such that

$$
z^{\prime \prime}\left(T, \lambda^{*}\right)>0, \quad z^{\prime \prime}\left(T, \lambda^{* *}\right)<0
$$

and therefore there exists $\lambda^{*} \in\left(\lambda_{3}, \lambda_{1}\right): z^{\prime \prime}\left(T, \lambda^{*}\right)=0$, i.e.,

$$
y^{\prime \prime}\left(T, \lambda^{*}\right)=\xi^{\prime \prime}(T)=B \text {. }
$$

In a similar way we get that there exists $\lambda^{\text {an }} \in\left(\lambda_{5}, \lambda_{3}\right)$ :

$$
y^{\prime \prime}\left(T, \lambda^{2+\infty}\right)=\xi^{\prime \prime}(T)=B .
$$

Step by step we get that there exists at least

(1) $(m-1) / 2$ solutions $y\left(t, \lambda^{*}\right), \lambda^{*} \in\left(\lambda_{r+2}, \lambda_{r}\right), r=1,3,5, \ldots, m-2$, if $m$ is odd;

(2) $(m-2) / 2$ solutions $y\left(t, \lambda^{*}\right), \lambda^{*} \in\left(\lambda_{r+2}, \lambda_{r}\right), r=1,3,5, \ldots, m-3$, if $m$ is even.

Including the solution $\xi$ completes the proof.

Corollary. Let $f$ be bounded on $R$; i.e., let there exist $M>0$ such that $|f(y)| \leq$ $M$ for $y \in R$. Let $f(0)=0$ and $\partial f(0) / \partial y<0$. Then the $B V P$

$$
\begin{aligned}
& y^{\prime \prime \prime}=f(y), \\
& y(0)=0, \quad y^{\prime}(0)=0, \\
& y^{\prime \prime}(T)=0
\end{aligned}
$$

has at least

$$
\begin{array}{ll}
\frac{m-1}{2}+1 \text { solutions } & \text { if } m \text { is odd, } \\
\frac{m-2}{2}+1 \text { solutions } & \text { if } m \text { is even }
\end{array}
$$

on $[0, T]$, for $T \geq 2(m \pi+2 \pi / 3) / \sqrt{3} \sqrt[3]{-\partial f(0) / \partial y}$, where $m \geq 3$ is a natural number.

Proof. We see that the function $\xi \equiv 0$ is the solution of $\left(1_{1}\right),\left(2^{\prime}\right),\left(3^{\prime}\right)$. The following problem

$$
\begin{gathered}
z^{\prime \prime \prime}=\frac{\partial f}{\partial y}(0) z, \\
z(0)=0, \quad z^{\prime}(0)=0, \quad z^{\prime \prime}(0)=1,
\end{gathered}
$$

is the corresponding variational problem. Denote by $L=\frac{1}{2} \sqrt[3]{-\partial f(0) / \partial y}$. Then

$$
\begin{aligned}
& z_{1}(t)=e^{-2 L t}, \\
& z_{2}(t)=e^{L T} \cos (\sqrt{3} L t), \\
& z_{3}(t)=e^{L t} \sin (\sqrt{3} L t)
\end{aligned}
$$

is the fundamental system of solutions of $\left(4_{1}\right)$ and the solution

$$
z(t)=\frac{1}{12 L^{2}} e^{-2 L t}-\frac{1}{12 L^{2}} e^{L t} \cos (\sqrt{3} L t)+\frac{\sqrt{3}}{12 L^{2}} e^{L t} \sin (\sqrt{3} L t)
$$


satisfies the initial conditions (5). Now we will investigate the zeros of $z$. Let

$$
\frac{1}{12 L^{2}} e^{-2 L t}-\frac{1}{12 L^{2}} e^{L t} \cos (\sqrt{3} L t)+\frac{\sqrt{3}}{12 L^{2}} e^{L t} \sin (\sqrt{3} L t)=0,
$$

i.e.,

Since

$$
\frac{1}{12 L^{2}} e^{-2 L t}=\frac{1}{12 L^{2}} e^{L t}[\cos (\sqrt{3} L t)-\sqrt{3} \sin (\sqrt{3} L t)]
$$

$$
\cos (\sqrt{3} L t)-\sqrt{3} \sin (\sqrt{3} L t)=2 \sin \left(\sqrt{3} L t+\frac{5 \pi}{6}\right)
$$

we have

$$
\frac{1}{12 L^{2}} e^{-2 L t}=\frac{1}{6 L^{2}} e^{L t} \sin \left(\sqrt{3} L t+\frac{5 \pi}{6}\right)
$$

i.e.

$$
\frac{1}{2} e^{-3 L t}=\sin \left(\sqrt{3} L t+\frac{5 \pi}{6}\right) .
$$

From the equality of two functions we get that equation has just one root in the interval

$$
\left(\frac{k \pi / 2-5 \pi / 6}{\sqrt{3} L}, \frac{(k+2) \pi / 2-5 \pi / 6}{\sqrt{3} L}\right), \quad k=3,5,7, \ldots, 2 m+1 .
$$

Hence the function $z(t)$ has at least $m$ zeros in $(0, T)$ and so the assumption (i) holds. Provided that $f$ is bounded, from Exercise $5.1[5$, p. 45] we have that every solution $y(t, \lambda)$ of $\left(1_{1}\right),\left(2^{\prime}\right),(8)$ can be extended to $[0, T]$ and so the assumption (iii) holds too.

Moreover, under the boundedness of $f$ we will verify (ii). Let $\eta$ be a solution of the initial value problem

$$
\begin{aligned}
& y^{\prime \prime \prime}=f(y), \\
& y(0)=0, \quad y^{\prime}(0)=0, \\
& y^{\prime \prime}(0)=\gamma
\end{aligned}
$$

where $\gamma>M T / 3$. Then

$$
\begin{aligned}
\eta(t) & =\eta(0)+\eta^{\prime}(0) t+\eta^{\prime \prime}(0) \frac{t^{2}}{2 !}+\int_{0}^{t} \frac{(t-s)^{2}}{2 !} f(\eta(s)) d s \\
& =\gamma \frac{t^{2}}{2 !}+\int_{0}^{t} \frac{(t-s)^{2}}{2 !} f(\eta(s)) d s \geq \gamma \frac{t^{2}}{2 !}-M \frac{t^{3}}{3 !} \\
& =\frac{t^{2}}{2}\left(\gamma-M \frac{t}{3}\right)>\frac{t^{2}}{2}\left(\gamma-M \frac{T}{3}\right)>0
\end{aligned}
$$

and hence $\eta(t)>\xi(t)$ on $(0, T]$. From Theorem 3 it follows that the proof is complete.

Example. The BVP

$$
\begin{aligned}
& y^{\prime \prime \prime}=\frac{8}{3 \sqrt{3}} \frac{-y}{\sqrt{1+y^{2}}}, \\
& y(0)=0, \quad y^{\prime}(0)=0, \\
& y^{\prime \prime}(T)=0
\end{aligned}
$$


has at least $(m-1) / 2+1$ solutions if $m$ is odd or $(m-2) / 2+1$ solutions if $m$ is even on $[0, T]$, for $T \geq m \pi+2 \pi / 3, m \geq 3$.

Proof. It follows from the corollary and the corresponding variational problem along the solution $\xi \equiv 0$ as follows

$$
\begin{gathered}
z^{\prime \prime \prime}=-\frac{8}{3 \sqrt{3}} z, \\
z(0)=0, \quad z^{\prime}(0)=0, \quad z^{\prime \prime}(0)=1 .
\end{gathered}
$$

It is obvious that $f$ is bounded and $\partial f(y) / \partial y=-1 /\left(1+y^{2}\right) \sqrt{1+y^{2}} \leq 0$.

\section{COMPLEMENTARY RESUlTS}

In this section we will find the conditions, which assure the accomplishment of assumptions (i), (ii), (iii) for the special case of BVP

$$
\begin{aligned}
& y^{\prime \prime \prime}=f(t, y), \\
& y(0)=A_{0}, \quad y^{\prime}(0)=A_{1}, \\
& y^{\prime \prime}(T)=B .
\end{aligned}
$$

Provided that $f$ is bounded, we will show that (i), (ii), (iii) can be achieved easily. For convenient reference we denote the associated homogeneous problem as follows

$$
\begin{aligned}
& y^{\prime \prime \prime}=0, \\
& y(0)=0, \quad y^{\prime}(0)=0, \\
& y^{\prime \prime}(T)=0 .
\end{aligned}
$$

The Green function $G(t, s)$ of $\left(1^{\prime}\right),\left(2^{\prime}\right),\left(3^{\prime}\right)$ is given by

$$
G(t, s)= \begin{cases}\frac{1}{2} s^{2}-s t, & 0 \leq s<t \leq T, \\ -\frac{1}{2} t^{2}, & 0 \leq t<s \leq T .\end{cases}
$$

It is easy to see that $G(t, s) \leq 0$ on $[0, T] \times[0, T]$. Now we deal with the assumption (i).

The existence of a solution $\xi$. There exist several types of theorems which ensure the existence of $\xi$. In [1] there are some growing conditions, conditions of potency and the condition of monotonicity and coercivity for the existence. The method of lower and upper solutions can also be available, and it is established on the fixed point theorem for monotone operators [6, Theorem 4.1]. The next theorem is in our interest:

Theorem 4 [11, p. 25]. Let us consider the BVP

$$
\begin{aligned}
& y^{\prime}=A(t) y+g(t, y), \quad(t, y) \in[b, a] \times R^{n} ; \\
& A_{1} y\left(t_{1}\right)+\cdots+A_{m} y\left(t_{m}\right)=\varphi\left(y\left(t_{1}\right), \ldots, y\left(t_{p}\right)\right),
\end{aligned}
$$

where $A(t)$ is an $n \times n$ matrix with integrable coefficients, $A_{s}(s=1,2, \ldots, m)$ are constant $n \times n$ matrices, $t_{s} \in[a, b]\left(s=1, \ldots, m_{0}\right), m_{0}=\max (m, p)$, $g(t, y):[a, b] \times R^{n} \rightarrow R^{n}$ satisfies Carathéodory conditions, $\varphi \in C\left(R^{n p}, R^{n}\right)$. 
Furthermore, let there exist $q \in L([a, b])$ and $\gamma>0$ such that

$$
\begin{array}{cc}
|g(t, y)|<q(t), & (t, y) \in[a, b] \times R^{n}, \\
\left|\varphi\left(u_{1}, \ldots, u_{p}\right)\right|<\gamma & \forall\left(u_{1}, \ldots, u_{p}\right) \in R^{n p} .
\end{array}
$$

Let the associated homogeneous $B V P$

$$
y^{\prime}=A(t) y, \quad A_{1} y\left(t_{1}\right)+\cdots+A_{m}(y)\left(t_{m}\right)=0
$$

have only trivial solution.

Then BVP (1.1), (1.2) has a solution.

Corollary. Let $f \in C([0, T] \times R, R)$ be bounded; i.e., let there exist $M>0$ such that $|f(t, y)| \leq M$ for $(t, y) \in[0, T] \times R$. Then BVP $\left(1_{2}\right)$, (2), (3) has a solution.

Proof. By the substitution $y_{1}=y$ the problem $\left(1_{2}\right),(2),(3)$ can be transformed to the problem of the type (1.1), (1.2) as follows:

$$
\begin{gathered}
\left(\begin{array}{l}
y_{1}^{\prime} \\
y_{2}^{\prime} \\
y_{3}^{\prime}
\end{array}\right)=\left(\begin{array}{lll}
0 & 1 & 0 \\
0 & 0 & 1 \\
0 & 0 & 0
\end{array}\right)\left(\begin{array}{l}
y_{1} \\
y_{2} \\
y_{3}
\end{array}\right)+\left(\begin{array}{c}
0 \\
0 \\
f\left(t, y_{1}\right)
\end{array}\right), \\
\left(\begin{array}{lll}
1 & 0 & 0 \\
0 & 1 & 0 \\
0 & 0 & 0
\end{array}\right)\left(\begin{array}{l}
y_{1}(0) \\
y_{2}(0) \\
y_{3}(0)
\end{array}\right)+\left(\begin{array}{lll}
0 & 0 & 0 \\
0 & 0 & 0 \\
0 & 0 & 1
\end{array}\right)\left(\begin{array}{l}
y_{1}(T) \\
y_{2}(T) \\
y_{3}(T)
\end{array}\right)=\left(\begin{array}{l}
A_{0} \\
A_{1} \\
B
\end{array}\right) .
\end{gathered}
$$

Let us denote

$$
\begin{array}{ccc}
\vec{y}=\left(\begin{array}{l}
y_{1} \\
y_{2} \\
y_{3}
\end{array}\right), & g(t, \vec{y})=\left(\begin{array}{c}
0 \\
0 \\
f\left(t, y_{1}\right)
\end{array}\right), & \varphi=\left(\begin{array}{l}
A_{0} \\
A_{1} \\
B
\end{array}\right), \\
A=\left(\begin{array}{lll}
0 & 1 & 0 \\
0 & 0 & 1 \\
0 & 0 & 0
\end{array}\right), & A_{1}=\left(\begin{array}{lll}
1 & 0 & 0 \\
0 & 1 & 0 \\
0 & 0 & 0
\end{array}\right), & A_{2}=\left(\begin{array}{lll}
0 & 0 & 0 \\
0 & 0 & 0 \\
0 & 0 & 1
\end{array}\right) .
\end{array}
$$

So the BVP $\left(1_{2}\right),(2),(3)$ is equal to the system

$$
\begin{gathered}
\vec{y}=A \vec{y}+g(t, \vec{y}), \\
A_{1} \vec{y}(0)+A_{2} \vec{y}(T)=\varphi .
\end{gathered}
$$

Since $f$ is the continuous function on $[0, T] \times R$, the function $g$ is continuous on $[0, T] \times R^{3}$. Further, $|f(t, y)| \leq M$ and thus $\|g(t, \vec{y})\| \leq M$ for $(t, \vec{y}) \in$ $[0, T] \times R^{3}$, where $\|\cdot\|$ is Euclidean norm in $R^{3}$. So in (1.3) it is sufficient to put $q \equiv M+1$. Since $\varphi$ is constant, the assumption (1:4) holds too. The associated homogeneous BVP (1.5), (1.6) has only trivial solution. Hence from Theorem 4 we get that there exists a solution $\xi$ of $\left(1_{2}\right),(2),(3)$.

Lemma 5. Let $f \in C([0, T] \times R, R)$ be bounded, i.e., let there exist $M>0$ such that $|f(t, y)| \leq M$ for $(t, y) \in[0, T] \times R$. Then for every solution $y$ of $\left(1_{2}\right),(2),(3)$ it holds that

$$
-M \frac{t^{2}}{3 !}(3 T-t)+P(t) \leq y(t) \leq M \frac{t^{2}}{3 !}(3 T-t)+P(t),
$$

where $P(t)=\frac{1}{2} B t^{2}+A_{1} t+A_{0}$. 
Proof. The BVP $\left(1_{2}\right),(2),(3)$ is equivalent with the integral equation

$$
y(t)=\int_{0}^{T} G(t, s) f(s, y(s)) d s+P(t),
$$

where $G(t, s)$ is Green function. Further,

$$
\left|\int_{0}^{T} G(t, s) f(s, y(s)) d s\right| \leq \int_{0}^{T}|G(t, s)| \cdot M d s=M \frac{t^{2}}{3 !}(3 T-t) .
$$

Similarly,

$$
-M \frac{t^{2}}{3 !}(3 T-t) \leq\left|\int_{0}^{T} G(t, s) f(s, y(s)) d s\right| .
$$

Hence the proof is complete.

The number of zeros of solution for the linear differential equations of the third order. Later in Theorem 7 we will need the following theorem from which we can derive the index $m$ of a solution $\xi$. Let us consider two differential equations

$$
\begin{aligned}
& z^{\prime \prime \prime}+b(t) z=0, \\
& z^{\prime \prime \prime}+B(t) z=0,
\end{aligned}
$$

where $B(t), b(t)$ are continuous functions on $\left[t_{0}, T\right]$ such that $B(t) \geq b(t)>$ 0 . Since these equations are linear ones, their solutions are defined and continuous functions on the whole interval $\left[t_{0}, T\right]$.

Theorem 5 [9]. Let every solution $z_{1}$ of (I) with a double zero at any point have another zero such that the distance between these zeros is less than or equal to a number $d$. Then every solution $z_{2}$ of (II) with the double zero at a point $t_{0}$ has zeros such that the distance between its two consecutive zeros is less than or equal to $d$.

Corollary. Let every solution $z_{1}$ of $(\mathrm{I})$ with a double zero at any point has another zero such that the distance between these zeros is less than or equal to a number $d$. Then the solution $z_{2}(t)$ of (II) with the double zero at a point $t_{0}$ has at least $m$ zeros in the interval $\left(t_{0}, t_{0}+m d\right]$.

Extendibility. The extendibility of solutions of initial value problems $\left(1_{2}\right)$, (2), (8) follows from Exercise 5.1 [5, p. 45]:

Theorem 6. Let $f \in C([0, T] \times R, R)$ be bounded. Then every solution of $\left(1_{2}\right)$, (2), (8) can be extended on $[0, T]$.

Main result. Now we connect the results of this section with the main Theorem 3 in $\S 2$.

Theorem 7. Let $f \in C([0, T] \times R, R)$ be bounded, i.e., let there exist $M>0$ such that $|f(t, y)| \leq M$ for $(t, y) \in[0, T] \times R$. Let

$$
\begin{aligned}
K_{M}=\{ & u \in C^{3}([0, T], R): \\
& \left.-M \frac{t^{2}}{3 !}(3 T-t)+P(t) \leq u(t) \leq M \frac{t^{2}}{3 !}(3 T-t)+P(t), t \in[0, T]\right\},
\end{aligned}
$$


where $P(t)=\frac{1}{2} B t^{2}+A_{1} t+A_{0}$. Furthermore, let $\mu>0$ be such that

$$
\frac{\partial f}{\partial y}(t, u(t)) \leq-\mu \quad \text { for } u \in K_{M} .
$$

Then the assumptions (i), (ii), (iii) hold and so the BVP (12), (2), (3) has at least

$$
\begin{aligned}
& \frac{m-1}{2}+1 \text { solutions } \quad \text { if } m \text { is odd, } \\
& \frac{m-2}{2}+1 \text { solutions } \quad \text { if } m \text { is even }
\end{aligned}
$$

on $[0, T]$, for $T \geq 2(m \pi+2 \pi / 3) / \sqrt{3} \sqrt[3]{\mu}$, where $m \geq 3$ is a natural number. Proof. From the Corollary of Theorem 4 and Lemma 5 we have the existence of a solution $\xi$ of $\left(1_{2}\right),(2)$, (3) such that $\xi \in K_{M}$. Therefore

$$
\frac{\partial f}{\partial y}(t, \xi(t)) \leq-\mu \quad \text { for } t \in[0, T] .
$$

The following problem

$$
\begin{gathered}
z^{\prime \prime \prime}-\frac{\partial f}{\partial y}(t, \xi(t)) z=0, \\
z(0)=0, \quad z^{\prime}(0)=0, \quad z^{\prime \prime}(0)=1,
\end{gathered}
$$

is the corresponding variational problem. The problem$$
z^{\prime \prime \prime}+\mu z=0,
$$$$
z(0)=0, \quad z^{\prime}(0)=0, \quad z^{\prime \prime}(0)=1,
$$

is the minorant problem for the problem $\left(4_{2}\right),(5)$. Since $\mu$ is the constant in the equation $\left(4_{\mu}\right)$, then the distance of zeros of a solution $z_{\mu}$ of $\left(4_{\mu}\right),(5)$ does not depend on $t$. Denote $L=\frac{1}{2} \sqrt[3]{\mu}$. Then

$$
z_{\mu}(t)=\frac{1}{12 L^{2}} e^{-2 L t}-\frac{1}{12 L^{2}} e^{L t} \cos (\sqrt{3} L t)+\frac{\sqrt{3}}{12 L^{2}} e^{L t} \sin (\sqrt{3} L t)
$$

is the solution of $\left(4_{\mu}\right),(5)$. Similarly the proof of corollary of Theorem 3 we get that the function $z_{\mu}(t)$ has at least $m$ zeros in $(0, T)$. From the corollary of Theorem 5 we have that the solution $z(t)$ of $\left(4_{2}\right),(5)$ has at least $m$ zeros in $(0, T)$, i.e. the solution $\xi$ of $\left(1_{2}\right),(2),(3)$ has the index $m$ and so assumption (i) holds.

From Theorem 6 we have that every solution $y(t, \lambda)$ of $\left(1_{2}\right),(2),(8)$ can be extended to $[0, T]$ and so assumption (iii) holds. Under the boundedness of $f$ we will show the existence of $\eta$ of $\left(1_{2}\right),(2)$ satisfying assumption (ii). Let

$$
\eta^{\prime \prime}(0)=\xi^{\prime \prime}(0)+\gamma
$$

for some appropriate $\gamma>0$. Then

$$
\begin{aligned}
\eta(t) & =\eta(0)+\eta^{\prime}(0) t+\eta^{\prime \prime}(0) \frac{t^{2}}{2 !}+\int_{0}^{t} \frac{(t-s)^{2}}{2 !} f(s, \eta(s)) d s \\
& =A_{0}+A_{1} t+\left(\xi^{\prime \prime}(0)+\gamma\right) \frac{t^{2}}{2 !}+\int_{0}^{t} \frac{(t-s)^{2}}{2 !} f(s, \eta(s)) d s
\end{aligned}
$$


and

$$
\begin{aligned}
\xi(t) & =\xi(0)+\xi^{\prime}(0) t+\xi^{\prime \prime}(0) \frac{t^{2}}{2 !}+\int_{0}^{t} \frac{(t-s)^{2}}{2 !} f(s, \xi(s)) d s \\
& =A_{0}+A_{1} t+\xi^{\prime \prime}(0) \frac{t^{2}}{2 !}+\int_{0}^{t} \frac{(t-s)^{2}}{2 !} f(s, \xi(s)) d s
\end{aligned}
$$

for $t \in[0, T]$. Hence we have

$$
\begin{aligned}
\eta(t)-\xi(t) & =\gamma \frac{t^{2}}{2 !}+\int_{0}^{t} \frac{(t-s)^{2}}{2 !} f(s, \eta(s)) d s-\int_{0}^{t} \frac{(t-s)^{2}}{2 !} f(s, \xi(s)) d s \\
& \geq \gamma \frac{t^{2}}{2 !}-M \int_{0}^{t} \frac{(t-s)^{2}}{2 !} d s-k_{1} \int_{0}^{t} \frac{(t-s)^{2}}{2 !} d s,
\end{aligned}
$$

where $t \in[0, T]$ and $k_{1}>0$ is a constant such that $|f(t, u)| \leq k_{1}$ for $u \in K_{M}$, i.e., $|f(t, \xi)| \leq k_{1}$. Thus

$$
\begin{aligned}
\eta(t)-\xi(t) & \geq \gamma \frac{t^{2}}{2 !}-M \frac{t^{3}}{3 !}-k_{1} \frac{t^{3}}{3 !} \\
& =\frac{t^{2}}{2 !}\left(\gamma-M \frac{t}{3}-k_{1} \frac{t}{3}\right) .
\end{aligned}
$$

For

$$
\gamma-M \frac{t}{3}-k_{1} \frac{t}{3}>0
$$

we get $\eta(t)>\xi(t), t \in(0, T]$. So if

$$
\begin{aligned}
& \gamma-M \frac{t}{3}-k_{1} \frac{t}{3}>\gamma-M \frac{T}{3}-k_{1} \frac{T}{3}>0 \\
& \quad \Rightarrow \gamma>M \frac{T}{3}+k_{1} \frac{T}{3}=\frac{T}{3}\left(M+k_{1}\right)>0,
\end{aligned}
$$

then the assumptions (ii) holds. Hence from Theorem 3 it follows that the proof is complete.

\section{REFERENCES}

1. S. Fučík and A. Kufner, Nonlinear differential equations, SNTL, Prague, 1978. (Czech)

2. M. Greguš and M. Gera, Some results in the theory of a third-order linear differential equation, Ann. Polon. Math. 42 (1983), 93-102.

3. M. Greguš, M. Švec, and V. Šeda, Ordinary differential equations, Alfa, Bratislava, 1985. (Slovak)

4. M. Greguš, Linear differential equation of the third order, Veda, Bratislava, 1981. (Slovak)

5. P. Hartman, Ordinary differential equations, Wiley, New York, London, and Sydney, 1964. (Russian translation)

6. M. A. Krasnoselskii, Positive solutions of operators equations, Gosud, Moscow, 1962. (Russian)

7. B. N. Petrov, V. Yu. Rutkovskii, and S. D. Zemlyakov, Adaptive coordinate-parameter control of flying apparatuses, control in cosmic space, vol. 1, Nauka, Moscow, 1972. (Russian)

8. V. Rocard, Dynamique générale des vibrations, Masson, Paris, 1949. (French)

9. E. Rovderová, The number of solutions of two point nonlinear boundary value problems, Dissertation Thesis, 1993. 
10. E. Sadyrbaev, About the number of solutions of the two point boundary value problem, Latvian Math. Ann. 32 (1988), 37-41. (Russian)

11. N. I. Vasiliev and Ju. A. Klokov, The elements of theory of boundary value problems for ordinary differential equations, Zinatne, Riga, 1978. (Russian)

Department of Mathematics, Faculty of Chemical Technology, Slovak Technical UNIVERSITY, RADLINSKÉHO 9, 81237 BRATISLAVA, SLOVAKIA

E-mail address: rovder@cvt.stuba.sk 